\title{
Tree state category identification for boreal area conifers using global features estimation by fuzzy logic approach
}

\author{
A S Pyataev ${ }^{1,2}$, A Y Redkin ${ }^{2}$ and A V Pyataeva ${ }^{1,3}$ \\ ${ }^{1}$ Reshetnev Siberian State University of Science and Technology, Krasnoyarsky Rabochy Av., \\ 31, Krasnoyarsk, Russia, 660037 \\ ${ }^{2}$ Brunch of FBI «Russian Centre of Forest Health»- Centre of Forest Health of Krasnoyarsk \\ Krai, Akademgorodok, 50A, building 2, Krasnoyarsk, Russia, 660036 \\ ${ }^{3}$ Siberian Federal University, Svobodny pr., 79, Krasnoyarsk, Russia, 660041 \\ e-mail: anna4u@list.ru
}

\begin{abstract}
Tree state category identification allows forecasting forest development in the surveyed area. Tree state category determination process based on global features is subjective and uses concepts such as the degree of density of the crown, the degree of drying of branches, the fall of the bark, the color of the needles, etc. For global features estimation, fuzzy logic is used. To formalize these subjective concepts, linguistic variables and their terms were extracted. The characteristic functions describing the terms were piecewise linear and in this work were approximated by Gaussian functions. Such an approach in conjunction with image processing algorithms that allows to search objects on images or correct images obtained for example from unmanned aerial vehicles could be the basis of a system for automatically determining the forest plantations health state and improve the inspection quality. The study was conducted for coniferous species of the boreal zone. The mathematical model built in this work allows reducing the cost of automation of calculations related to the processing of the data obtained by forest pathological surveys, despite the fact that the accuracy value of fuzzy classification after the approximation of the membership functions remained at the same level.
\end{abstract}

\section{Introduction}

Determining the tree state category by a set of visual marks is widely used in forest health diagnostics to estimate sanitary conditions of forest plantations. This estimation based on forest health diagnostics results allows to planning forest protection events system, which contains preventive, protective and exterminatory actions. Tree sanitary condition estimation correctness depends on forest health engineer experience and qualification. Forest health engineer such estimation made uses subjective concepts such as crown density, conifer shades, defoliation grade, etc.

In Russia, eleven categories of tree sanitary status are allocated. This is defined by the Russian Federation Government Resolution No. 607 of May 20, 2017 "On the Rules of Sanitary Safety in Forests". The paper considers the signs of only the first six tree state categories, since the last five categories are beyond the scope of the research interests, because they characterize fallen trees. Fallen trees include windfall, windbreak, snowbreak and emergency trees. The difficulty of the tree state category determination depends on fuzzy, subjective concepts, on the basis of which the forest health engineer makes conclusions about assigning the tree to one or another category. 
To formalize such subjective concepts in our approach we use fuzzy logic. Nowadays, fuzzy logic is widely used in various fields of human activity: from environmental issues [1] to applied problems of disaster impact assessment [2]. In forest development tasks, fuzzy logic is used for estimation of the plants growth potential [3], for forest cuttings types classifications [4], in problems of forest fire forecasting and detection [5, 6], in problems of forest protection [7].

Tree state category division based on symptom analyses, which could be separated by local and global. The presence of hollows, conifer damage, burns, etc. could be classified as local symptoms. At the same time, crown density, conifer shades, growth size, defoliation grade, shrinking branches grade and bark falling could be classified as global symptoms. Global symptoms description subjectiveness generates difficulties and dissensions when tree state categories are being estimated. Global symptoms determination approach based on fuzzy logic designed to fix this problem. Such an approach in conjunction with image processing algorithms that allows to search objects on images $[8,9]$ or correct images [10] obtained for example from unmanned aerial vehicles could be the basis of a system for automatically determining the forest plantations health state.

\section{Theoretical research}

In paper [11] were extracted linguistic variables and terms, shown in table 1, to formalize subjective concepts of global symptoms determination. Terms domain and definition are built in cooperation with Krasnoyarsk centre of forest health specialists.

Table 1. Linguistic variables and its terms.

\begin{tabular}{|l|l|}
\hline \multicolumn{1}{|c|}{ Linguistic variable } & \multicolumn{1}{c|}{ Terms } \\
\hline crown density & rich, sparse, openwork, very openwork \\
\hline growth & normal, reduced, small, very small or nonexistent \\
\hline shrinking branches grade & $\begin{array}{l}\text { nonexistent, dry unitary branches, crown drying out less than } 2 / 3 \\
\text { volume, crown drying out more than 2/3 volume }\end{array}$ \\
\hline bark falling & nonexistent, partial, full \\
\hline conifer shade & $\begin{array}{l}\text { green, light green, yellow green, flavescent, yellow, red-brown, } \\
\text { gray }\end{array}$ \\
\hline
\end{tabular}

The characteristic functions, describes terms, were piecewise linear (figure 1) and specified in tabular form. Such form of function description makes it difficult to automate calculations. One of the problem solutions is to approximate piecewise linear functions by a smooth function.

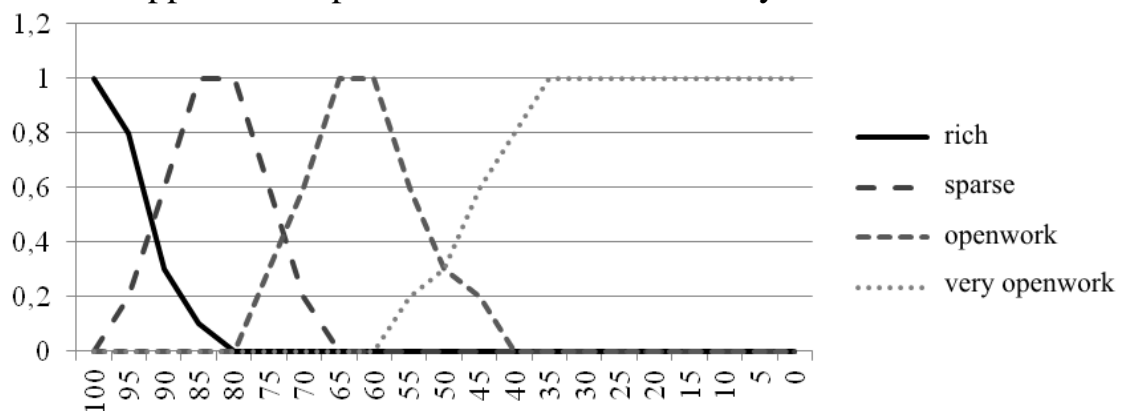

Figure 1. The linguistic variable «crown density» terms graphics.

Approximation methods are actively used in different fields of study to solve various problems. In paper [12] derived a new finite dimensional semidiscrete approximation scheme for systems of linear neutral delay-differential equations and proved convergence results. Paper [13] considers a fuzzy data approximation method defined at a 3D fuzzy data set. A fuzzy smoothing bicubic spline approximation for a given fuzzy data set is defined and the approximation error using similarity measures of fuzzy numbers is estimated. Study [14] considers the use of the convolution method for constructing approximations comprising fuzzy number sequences with useful properties for a general fuzzy number. It shows that this convolution method can generate differentiable approximations in finite steps for fuzzy numbers with finite non-differentiable points. Paper [15] considers a smoothing 
method of a set of points to be approximated from a given boundary value problem for the modified Helmholtz equation. In paper [16] the context interpolation algorithms for multidimensional signals in the compression problem are investigated. Interpolation algorithm based on context modeling for the hierarchical compression method for arbitrary dimension signals is proposed. The algorithm is based on optimizing parameters of the interpolating function in a local neighborhood of the interpolated sample. Wherein locally optimal parameters, which, were found for more sparse scale signal levels, are used to interpolate samples of less sparse scale signal levels.

In our paper, we carried out a spline approximation of previously corrected characteristic functions with Gauss functions. The original piecewise linear functions were presented in tabular form. The aim of the approximation made was to find functions that are as close as possible to the original piecewise linear functions, wherein the functions obtained should keep classification quality.

The approximation functions received could be used not only for pine, but for every boreal coniferous species. Gauss function spline approximation result for linguistic variable «crown density» terms graphics shown in table 2.

Table 2. Linguistic variable «crown density» characteristic functions

\begin{tabular}{|l|c|}
\hline Term & Characteristic functions \\
\hline Rich & $f(x)=\left\{\begin{array}{r}1 / \exp \left[(x-97)^{2} / 32\right], x \leq 97 \\
1, x>97\end{array}\right.$ \\
\hline Openwork & $f(x)=\left\{\begin{array}{r}1 / \exp \left[(x-82)^{2} / 50\right], x \leq 82 \\
1, x \in(82,87) \\
1 / \exp \left[(x-87)^{2} / 32\right], x \geq 87\end{array}\right.$ \\
\hline Very openwork & $f(x)=\left\{\begin{array}{r}1 / \exp \left[(x-55)^{2} / 50\right], x \leq 55 \\
1, x \in(55,70) \\
1 / \exp \left[(x-70)^{2} / 50\right], x \geq 70\end{array}\right.$ \\
\hline
\end{tabular}

Figure 2 considers approximated graphics for each term. X-axis contains crown density percent and $\mathrm{Y}$-axis describes assurance degree.

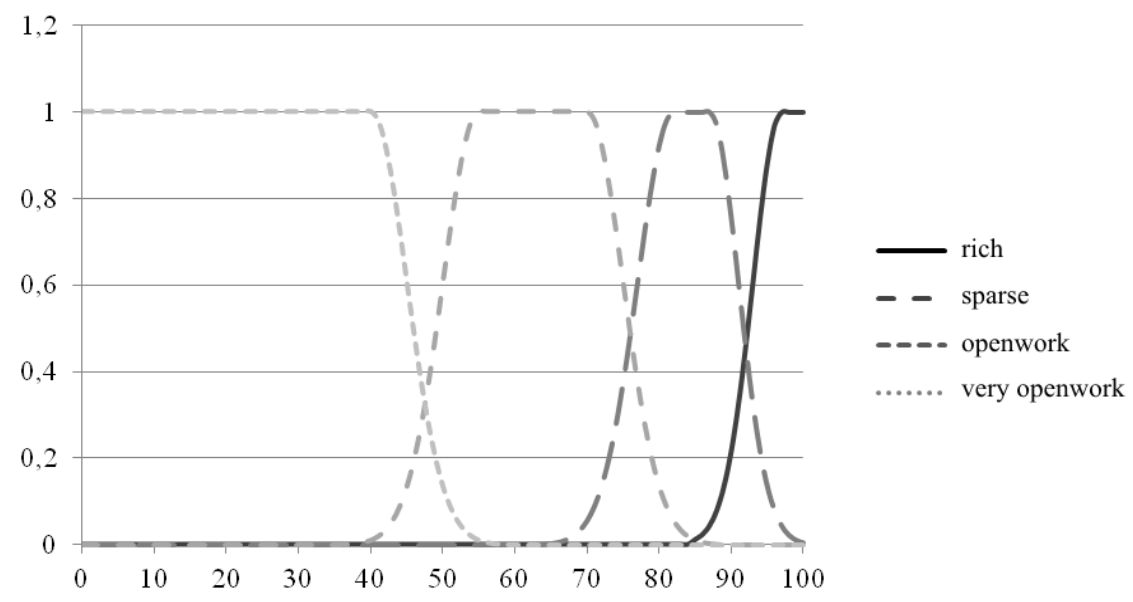

Figure 2. The linguistic variable «crown density» approximated terms graphics. 
Characteristic functions approximation made similarly for the rest of linguistic variables. Figure 3 shows previously corrected piecewise linear terms graphics and approximated terms graphics:

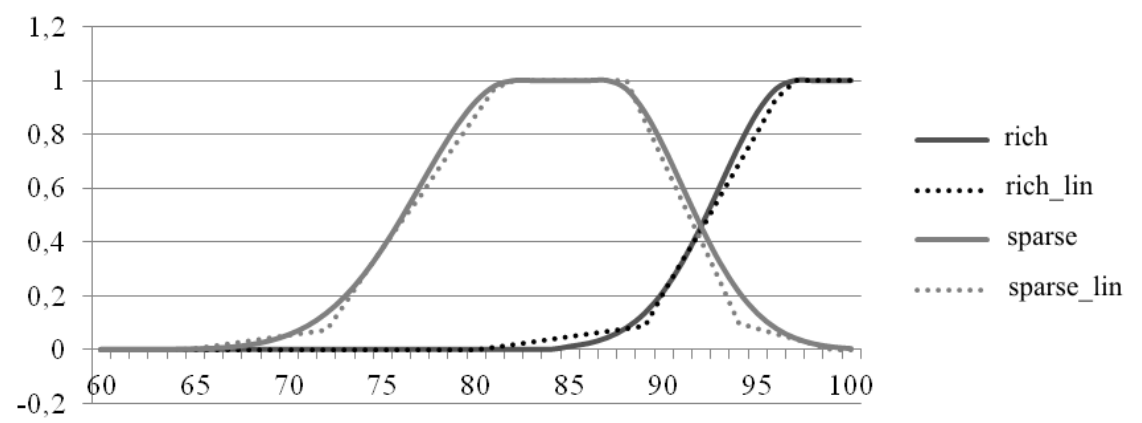

Figure 3. Comparison of the piecewise linear and approximated graphics for terms.

Fuzzy rules knowledgebase has been produced on linguistic variables characteristic functions. Fuzzy rules examples that allow making a conclusion about the state category of the surveyed tree are given below:

- IF («crown density» = «rich») AND («growth» = «normal») AND («shrinking branches grade $»=\langle 0 »)$ THEN $(«$ state $»=\langle$ healthy») .

- IF («crown density» = «openwork») AND («growth» = «small») AND («shrinking branches grade» $=\langle$ from $10 \%$ to $65 \% »)$ THEN («state» $=\langle$ sseverely weakened $»)$.

According to the approximation results, there was no significant change neither in the clear output value, nor the degree of confidence in the introduction of fuzziness, nor the triggering force of the rules, nor the type of output figure. The reason for this was similarity of the approximating functions and the original piecewise linear functions, so the quality of the fuzzy classification has not been changed.

\section{Conclusion}

In this paper, a mathematical model is constructed allows describing subjective concepts that influence on the formation of a conclusion about the tree sanitary condition. A spline approximation of the initial characteristic piecewise linear functions by Gauss functions was performed to solve the problem of estimating the category of the coniferous trees of the boreal zone according to global features based on fuzzy logic. The mathematical model built in this work allows reducing the cost of automation of calculations related to the processing of the data obtained by forest pathological surveys, despite the fact that the accuracy value of fuzzy classification after the approximation of the membership functions remained at the same level.

\section{References}

[1] Saadoud D, Hassani M, Peinado José Martin F and Saïd Guettouche M 2018 Application of fuzzy logic approach for wind erosion hazard mapping in Laghouat region (Algeria) using remote sensing and GIS Aeolian Research 32 24-34

[2] Ahmed S, Abed M and Mebarki A 2018 Post-earthquake assessment of buildings damage using fuzzy logic Engineering Structures 166 117-127

[3] Strnad D, Kohek Š and Kolmanič S 2018 Fuzzy modelling of growth potential in forest development simulation Ecological Informatics $\mathbf{4 8}$ 80-88

[4] Samec P, Caha J, Zapletal M, Tuček P, Cudlín P and Kučera M 2017 Discrimination between acute and chronic decline of Central European forests using map algebra of the growth condition and forest biomass fuzzy sets: A case study Science of The Total Environment 599600 899-909

[5] Garcia-Jimenez S, Jurio A, Pagola M, Miguel De L, Barrenechea E and Bustince H 2017 Forest fire detection: A fuzzy system approach based on overlap indices Applied Soft Computing $\mathbf{5 2}$ 834-842 
[6] Lin H, Liu X, Wang X and Liu Y 2018 A fuzzy inference and big data analysis algorithm for the prediction of forest fire based on rechargeable wireless sensor networks Sustainable Computing: Informatics and Systems 18 101-111

[7] Zeng X, Chen C, Liu A, Wei H, Zhang H, Huang G and Wu Y 2018 Planning a sustainable regional irrigated production and forest protection under land and water stresses with multiple uncertainties Journal of Cleaner Production 188 751-762

[8] Zakharov A A, Barinov A E, Zhiznyakov A L and Titov V S 2018 Object detection in images with a structural descriptor based on graphs Computer Optics 42(2) 283-290 DOI: 10.18287/2412-6179-2018-42-2-283-290

[9] Vizilter U V, Gorbatsevich V S, Vishnyakov B V and Sidyakin S V 2017 Object detection in images using morphlet descriptions Computer Optics 41(3) 406-411 DOI: 10.18287/2412-61792017-41-3-406-411

[10] Kozak A V, Steinberg B Y and Steinberg O B 2018 Fast restoration of a blurred image obtained by a horizontally rotating camera Computer Optics 42(6) 1046-1053 DOI: 10.18287/2412-61792018-42-6-1046-1053

[11] Pyataev A 2018 Tree state category identification for pine based on fuzzy logic by visual data Proc. Int. Conf. Regional problems of earth remote sensing (Krasnoyarsk)

[12] Fabiano R H and Catherine Payne 2018 Spline approximation for systems of linear neutral delay-differential equations Applied Mathematics and Computation 338 789-808

[13] Gonzalez P, Idais H, Pasadas M and Yasin M 2018 3D fuzzy data approximation by fuzzy smoothing bicubic splines Mathematics and Computers in Simulation

[14] Huang H, Congxin W, Jialian X and Dongxiao Z 2017 Approximation of fuzzy numbers using the convolution method Fuzzy Sets and Systems 310 14-46

[15] Kouibia A, Pasadas M, Reyah L and Akhrif R 2019 Approximation of surfaces by modified Helmholtz splines Journal of Computational and Applied Mathematics 350 262-273

[16] Gashnikov M V 2018 Interpolation based on context modeling for hierarchical compression of multidimensional signals Computer Optics 42(3) 468-475 DOI: 10.18287/2412-6179-2018-42$3-468-475$ 